\title{
Final Site Assembly and Testing of the Superconducting Toroidal Magnet for the CEBAF Large Acceptance Spectrometer (CLAS)
}

\author{
Alan J. Street, John S. H. Ross, Stephen M. Harrison, David M. Jenkins, Michel F. Mason, Richard J. Riggs, \\ Kevin D. Smith and Julian M. Wiatrzyk \\ Oxford Instruments Ltd., Old Station Way, Eynsham, Witney, Oxon OX8 1TL, UK \\ John E. O'Meara, Walter Tuzel and Doug Tilles \\ CEBAF, 12000 Jefferson Avenue, Newport News, VA23606, USA
}

\begin{abstract}
The design, manufacture and works testing of single coils has been reported at ASC, Chicago 1992 [1] and EPAC, London 1994 [2]. The assembly, commissioning and testing of the full six-coil toroidal spectrometer magnet is described and the results compared with guaranteed or expected values. The SURA contract number for this project is 91-C1882LD.
\end{abstract}

\section{INTRODUCTION}

The CLAS Torus magnet system consists of six, flat kidney-shaped coils connected in series and arranged around the beam line. The operating current is $3861 \mathrm{~A}$, producing a peak field of $3.5 \mathrm{~T}$.

Each coil has its own stainless steel vacuum case, but all are linked so that the whole system has a single vacuum. These cases are connected together directly at the high field end of the magnet, and at the other end are bolted onto a room temperature support ring. A number of carbon fibre tension ties link the outer profiles of the vacuum cases to ensure the integrity of the structure. A cryogenic Service Module interfaces with the CEBAF refrigerator, and controls the supply of cryogens to the coils via a circular manifold which is attached to the support ring.

The coils and the Service Module were tested individually at Oxford before shipping to the customer's facility in October 1994, but the manifold was untested, and the major components had never been assembled together.

The system was successfully installed between November 1994 and February 1995. It was then cooled from ambient temperature to $4.4 \mathrm{~K}$ and run to full field in both polarities.

\section{INSTALLATION}

The six coils were first assembled onto a temporary steel support structure, and then the positions of the vacuum cases were adjusted for optimum coil symmetry. The locations of the coils within the vacuum cases had been established by magnetic field measurements during works tests, and a

Manuscript received 12 June 1995. specially machined gauge block was used to take up inplane adjustments (up to $0.3 \mathrm{~mm}$ ) in the high field region. Circumferential symmetry of the vacuum cases was achieved by jacking from the support structure and by careful mechanical measurement.

The warm support ring and the carbon fibre ties between vacuum cases were attached. Gravitational loads were then transferred from the support structure to the ties: the loads on the ties were monitored using extensometers. The six coils were then raised $10 \mathrm{~mm}$ to the beam line and suspended from the two CEBAF supports. Finally, the temporary steelwork was removed.

The cryogenic manifold, which incorporates piping, insulation and instrumentation, could not be assembled until all six vacuum cases were in position. It was therefore built in the experimental hall onto the warm support ring, with all cryogenic and electrical connections made in situ. The Service Module, which had been shipped complete, was installed on the floor level above the magnet. Cryogenic and electrical connections - including the main power leads were made between the Service Module and the manifold before the sloping duct was built around them to link the vacuum spaces.

All of the necessary services - electrical power, instrumentation, cooling water and vacuum pump set - were then connected to the Service Module. The cryogenic supply lines from the refrigerator were welded on in a transition section through a vacuum break, so that the refrigerator and magnet vacuums remain entirely separate. The remaining cryogenic lines enter and leave through bayonets in the top plate.

\section{CoOL Down To $80 \mathrm{~K}$}

After purging to remove water and nitrogen from the helium circuits, the vacuum space was evacuated to $10^{-2}$ mbar. Cool down was then started by filling the liquid nitrogen storage vessel in the Service Module. This liquid was used to cool down the radiation screens and also to precool a supply of ambient temperature helium for cooling the coils. 
Liquid nitrogen and ambient temperature nitrogen gas were combined in a mixer to produce a stream at a controlled temperature which was supplied to the radiation screens. The temperature was ramped down automatically at a rate of around $2 \mathrm{~K} /$ hour so that the screens were cooled gently enough to avoid significant thermal stresses. Once the temperatures were all below $130 \mathrm{~K}$, liquid alone was supplied to achieve the operating temperature of $84 \mathrm{~K}$.

The coils were cooled in a similar way using helium. The coolant temperature was controlled by mixing gas which had been cooled to $84 \mathrm{~K}$ (by heat exchange with the liquid nitrogen in the Service Module) together with ambient temperature gas.

A total of eleven days were taken to cool the entire system to $80 \mathrm{~K}$, which was close to the original estimate of nine days. The delay was mainly due to a malfunction in the vacuum pump controller, which had to be replaced.

\section{COOL Down To $4.4 \mathrm{~K}$}

Once the coil temperatures were all close to $80 \mathrm{~K}$, the cold helium supply valve in the refrigerator was opened. With the helium supplied at $5 \mathrm{~K}$ and 2.8 ata the coils cooled rapidly, falling to under $10 \mathrm{~K}$ within a day. When $7 \mathrm{~K}$ had been reached in all six coils, the refrigerator supply valve was throttled so that helium liquefied in the transfer line to the Service Module, eventually flooding the magnet helium circuit and stabilising the temperature at $4.4 \mathrm{~K}$.

Because of the geometry of the cooling path, it is necessary for the helium supply to be supercritical: it is liquefied in the Service Module after flowing around the coils. The Joule-Thomson valve in the Service Module was therefore restricted as the supply pressure was increased until the cryogenic system reached its nominal stable operating condition.

\section{CRYOGENIC HEAT LOADS}

With the magnet fully cold, measurements were taken allowing the cryogenic heat loads to be calculated. The heat load to $4.4 \mathrm{~K}$ was $57 \mathrm{~W}$, and to $80 \mathrm{~K}$ approximately $250 \mathrm{~W}$. These values are comfortably within the guarantees of $110 \mathrm{~W}$ and $600 \mathrm{~W}$ respectively.

The current leads operated self-sufficiently, returning warm helium to the recovery system at a rate of $0.42 \mathrm{~g} / \mathrm{s}$. This rate of helium consumption is equivalent to $1.09 \mathrm{~W} / \mathrm{kA}$, and is well within the guaranteed efficiency.

\section{COLL ADJUSTMENT}

For good field homogeneity, and to keep the lateral magnetic forces to acceptably low levels, it is necessary for the coils to be positioned symmetrically to an accuracy within $\pm 1.5 \mathrm{~mm}$. For this reason, the out-of-plane coil supports were designed to be adjustable while the magnet is under vacuum and fully cold. The supports are also strain gauged so that the lateral forces can be monitored. Although the positions of the coils within the vacuum cases had been ascertained during works tests at Oxford with each coil vertical, some adjustment was inevitable, as the vacuum cases deflected slightly under their own weights when assembled into the torus.

When the cryogenic system was fully cold and stable, the power supply was switched on and the magnet was charged to a current of $1000 \mathrm{~A}$. The load indications from the seventy-two strain gauged supports were then evaluated and the coil positions inside the vacuum cases were adjusted. This procedure was repeated a total of twenty times until coil adjustments of just $0.2 \mathrm{~mm}$ were being made. The final adjustments were carried out after the current had been increased to $3500 \mathrm{~A}$ for better resolution.

\section{QUENCH}

The six coils had all been tested individually at Oxford to the full rated current of $3861 \mathrm{~A}$. Each had been quenched deliberately from full current and then re-energised: one only had experienced a training quench at $3300 \mathrm{~A}$ or $85 \%$ of rated current. It was intended to quench the torus from full current by temporarily disabling the power supply interlocks and closing off the cooling flow to the power leads. (Normally loss of lead coolant would cause the power supply to reduce the current to zero automatically.)

At the first attempt to run to full field Coil $\mathrm{E}$ (fifth in series from the power supply) quenched at $3800 \mathrm{~A}$, or $98.4 \%$ of rated current. The magnet features passive and active quench propagation to prevent the entire stored energy from being dissipated in a single coil. In this case the mean temperature reached in Coil E was $65 \mathrm{~K}$, while the other coils all rose to $55 \mathrm{~K}$. This indicates that Coil $\mathrm{E}$ received $25 \%$ of the stored magnetic energy, with the remaining $75 \%$ distributed evenly between the other five coils: the propagation system was therefore very effective.

The temperature rises were close to predicted values, and well within safe limits.

The quench relief valves lifted as required, venting the liberated helium into CEBAF's recovery system.

\section{RECOVERY AND FULL CURRENT TESTS}

When the magnet quenched, the cold helium supply and return valves were closed to protect the refrigerator. Once the system had stabilised the valves were opened to begin cooling again. When the coil temperatures had all fallen to $6.5 \mathrm{~K}$, the Joule-Thomson valve in the Service Module was throttled to cool by expansion until liquid began to collect: no action was required from the refrigerator.

The magnet was then run to full field in 2 hours and 18 minutes - significantly more quickly than the specified maximum time of 4 hours. After 25 minutes at full field the 
current was reduced to zero over a period of 90 minutes by ramping down the power supply. The polarity of the power supply was reversed and the magnet run to full field again.

\section{REFRIGERATOR FAILURE TEST}

The final test was to simulate a refrigerator failure while the magnet was at full field. The Service Module had been designed to have sufficient endurance to allow full discharge without quenching. The contract requirement was to discharge to $30 \%$ of rated current without quenching.

With the magnet current at $3861 \mathrm{~A}$, the refrigerator supply and return valves were closed. The power supply tripped into a fast discharge mode, and additional vent valves on the Service Module opened to compensate for the increased back pressure from the refrigerator.

It took an hour for the current to fall to zero. During this time, the highest coil temperature recorded by the instrumentation system was $5.2 \mathrm{~K}$ at a current of $2300 \mathrm{~A}$ : a considerable temperature margin. The magnet did not quench.

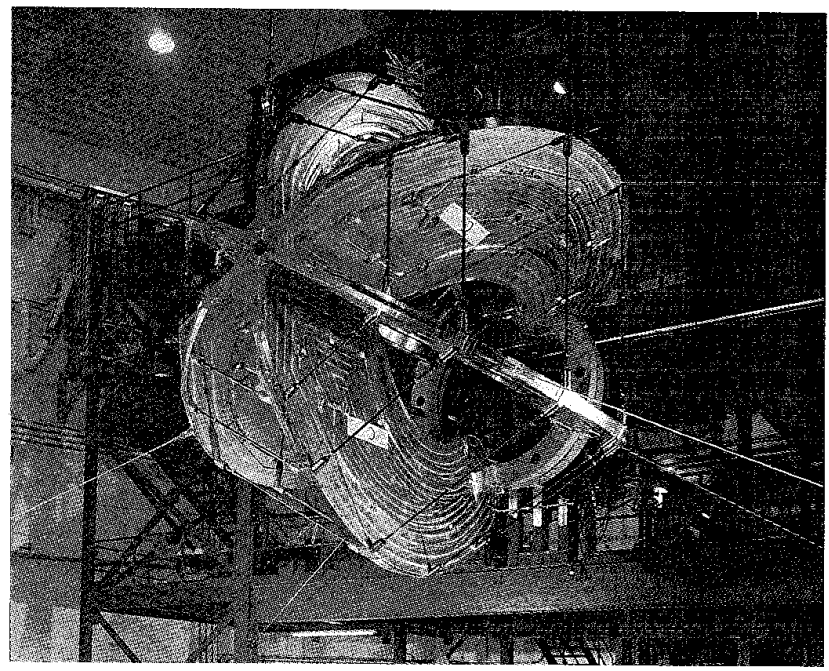

Fig. 1 . The fully assembled torus system installed at CEBAF.

\section{$\mathrm{X}$. CONClusions}

The installation and commissioning have been completed of the first commercially produced superconducting torus magnet of this scale. The final tests reported here were completed within one week of the contract target date.

\section{REFERENCES}

[1] J. S. H. Ross, K. D. Smith, A. J. Street, et al. "Development in the design of the superconducting toroidal magnet for the Continuous Electron Beam Accelerator Facility (CEBAF) large acceptance spectrometer", Applied Superconductivity Conference, August 1992.

[2] J. S. H. Ross, K. D. Smith, A. J. Street, et al. "Preliminary testing of coils for the superconducting toroidal magnet for the CEBAF large acceptance spectrometer (CLAS)", Cryogenics 1994 vol. 34 ICEC supplement, pp. 659-662, 1994. 